
\title{
28 Research Square \\ In-Situ Preparation of Novel Nanocomposites of PMMA and Ordered Mesoporous Carbon (FDU-15)
}

Gholamhossein Mohammadnezhad ( $\nabla$ mohammadnezhad@iut.ac.ir) https://orcid.org/0000-0003-1765-8063

Milad Okhovat

Tanin Fazel

Mohammad Dinari

\section{Research Article}

Keywords: PMMA, Ordered mesoporous carbon (FDU-15), KH-550, In-situ polymerization, Thermogravimetric analysis, Modification

Posted Date: February 7th, 2022

DOI: https://doi.org/10.21203/rs.3.rs-1320313/v1

License: (c) (i) This work is licensed under a Creative Commons Attribution 4.0 International License.

Read Full License 


\section{Abstract}

Ordered mesoporous carbons have recently been given significant attention because of their unique hexagonal structure, tunable large pore volumes, high thermal stability, and high surface-to-volume ratio. In this research paper, for achieving the maximum compatibility between the FDU-15 and poly(methyl methacrylate) (PMMA), $\mathrm{KH}-550$ was used as a modifying and coupling agent to facilitate the bond formations between the mesoporous carbon and PMMA with the help of ultrasonic irradiation. Nanocomposite (NC) films were produced via the in-situ polymerization method aligned with various amounts of modified FDU-15. Sample characterizations were done by Field-emission scanning electron microscope (FE-SEM), low angle X-ray diffraction (LXRD), Fourier transform infrared spectroscopy, transmission electron microscopy (TEM), and thermogravimetric analysis (TGA). The LXRD pattern proved that the modification process did not affect the hexagonal mesoporous structure of FDU-15. The PMMA-m-FDU $2 \mathrm{wt} \%$ showed the existence of some pores on its surface according to FE-SEM. The TEM image of the NC depicted a uniform dispersion of mesoporous carbon into the polymer matrix and also confirmed the hexagonal 2D structure of the mesopores. TGA data also confirmed that as the m-FDU-15 increases into the PMMA matrix, the higher thermal stability will achieve, and also the $T_{d}$ will increase to the higher temperatures.

\section{Introduction}

Poly(methyl methacrylate) (PMMA) is a synthetic thermoplastic polymer that is obtained by the additional polymerization process of methyl methacrylate (MMA). It shows unique properties such as being highly transparent, having very lightweight, and being resistant to expansions and contractions in hot and cool temperatures, respectively [1,2]. These properties make it a really good alternative for a typical glass. However, its application is not excluded from this and it is used in various industries such as resins and coatings or even in inks [3, 4]. It is also an inexpensive alternative for polycarbonates (PC) because not only the toxic bisphenol-A material does not exist in its structure, but also shows better characteristics such as polishability ability and laser cutting than those of PC $[5,6]$. The lack of the bisphenol-A in the structure of the PMMA makes it a good candidate in biomedical, and other applications. A series of NCs including the PMMA and Ti-Mo-Cu were synthesized to compensate for the mechanical weakness of porous Ti-Mo-Cu alloys in tissue engineering. The results showed a significant increase in the compressive strength of the newly obtained NCs in comparison with Ti-Mo-Cu alloys, which helped them to be appropriate for tissue engineering [7]. However, being susceptible to scratches and brittleness and showing low resistance to strong solvents in its pure state limit its usage in different applications [8-10]. Therefore, it must be combined with some fillers such as mesoporous organic material (MOM)s and ordered mesoporous carbon (OMC) to compensate for its drawbacks.

MOMs (size 2-50 nm) have been widely utilized as fillers for polymers because of their specific features. Their structure consists of the well-ordered arrays of uniform channels in the nano dimension, high mechanical and thermal stability, being inert to a variety of chemicals, high surface to the volume ratio and also, their large pore volume and being in a diversity of forms such as powders, foams, and fibers 
which make them widely used in applications such as adsorption of dyes and heavy metals in the contaminated water $[11,12]$.

$\mathrm{OMC}$ is a porous material with interconnected channels which can be fabricated by direct carbonization of organic-organic nanocomposites [13]. Like MOMs, OMCs also possess similar characteristics such as the high surface area to the volume ratio, significant chemical and thermal stability, and hydrophobicity. They are widely utilized in the adsorption applications like the adsorption of dyes and act as an adsorbent in hydrogen or carbon monoxide for gas storage purposes [14]. For example, a series of OMCs were prepared via the hard-templating method in a mesoporous silica template (KIT-6) and their application in the removal of AV90 dye was also examined. The results revealed the maximum removal of dye at $\mathrm{pH} 2$ and the structure of these materials did not alter after the adsorption process [15].

One of the most common OMCs used as a filler in NCs is FDU-15. FDU-15 possesses a 2-D hexagonal structure and hydrophobic nature having sizeable pore volumes $\left(0.65-0.85 \mathrm{~g} / \mathrm{cm}^{3}\right)$ with a high BET surface area between 650 to $1500 \mathrm{~m}^{2} / \mathrm{g}$. This structure makes it applicable in water remediation with hydrophobic nature $[14,16]$. A study has been done by Wu and his co-workers have shown that the removal capacity of alkaline earth metals increases with the amount of FDU-15. The results revealed that all the alkaline doped FDU-15 possessed significant adsorption capacity and the adsorption process occurred in either the conversion of nitrogen monoxide and oxygen to nitrogen dioxide and then to nitrite or direct oxidation of nitrogen monoxide to nitrite or nitrate [17].

However, there would be trouble using FDU-15 as the reinforcing agent in the polymer matrix. The hydrophobic nature of FDU-15 is not compatible with the polymer matrix and makes it difficult to produce homogenous NCs and finally leads to the accumulation of these mesopores into the matrix $[18,19]$. Therefore, they must be modified with some coupling agents such as 3-aminopropyltriethoxysilane (KH550 ) to decrease the agglomeration of mesoporous into the polymer matrix. Mohammadnezhad and his co-workers designed a series of NC films based on the PMMA and FDU-15 mesoporous using 3mercaptopropyl-trimethoxysilane as a modifying agent and studied their thermal stability and mechanical behavior. The result showed that by increasing the amount of FDU-15 mesoporous, the thermal stability and their mechanical behavior rise as well [20].

$\mathrm{KH}-550$ has been extensively used to make a bridge between inorganic fillers and the organic polymer matrix. On the one hand, the oxygen atom on its structure makes a covalent bond with the filler and on the other hand, the primary amine portion reacts with the organic function groups in the polymer matrix [21].

Ultrasonic irradiation as an efficient and green method has widely been exploited to achieve the maximum dispersion of fillers into the NCs. This helps to achieve the maximum interaction between polymer chains and nanoparticles become possible. In a study that was done by Okhovat and his coworker, a series of NCs based on chitosan-tragacanth blend reinforced with ZnO@Ag nanoparticles were built by the use of ultrasonic irradiation and they were used in bioactivity and antibacterial activity. The 
result showed that all the NCs are very compatible with hydroxyapatite and also, they showed an extreme antibacterial activity against both gram-negative and gram-positive bacteria [22].

In this project, several kinds of NCs were fabricated by the incorporation of different $(0.5,1,2$, and 3$)$ wt $\%$ of the FDU-15s modified with $\mathrm{KH}-550$ (m-FDU) into the PMMA matrix through the in-situ polymerization by the aid of ultrasonic irradiation. Various techniques and instruments were applied to characterize these NCs. Fourier transform infrared spectroscopy (FT-IR) by the aim of investigation of the functional groups, Low angle X-ray diffraction (LXRD) was utilized for the study of the crystallinity, field emission scanning electron microscopy (FE-SEM) for the morphological investigation, and transmission electron microscopy (TEM) to observe the shape and dispersion of m-FDUs into the polymer matrix were used. Moreover, investigation of the influences of these mesoporous structures on the thermal stability of the PMMA was examined by the thermogravimetric analysis (TGA).

\section{Experimental \\ 2.1. Materials}

All the materials including P123 (triblock poly(ethylene oxide)-poly(propylene oxide)-poly( ethylene oxide), $\left.\mathrm{Mw}=5.8 \times 10^{3}\right)$ 3-aminopropyltriethoxysilane $(\mathrm{KH}-550)$, benzoyl peroxide $(\mathrm{BP})$, methyl methacrylate $\left(M M A, M w=3.5 \times 10^{4} \mathrm{~g} \cdot \mathrm{mol}^{-1}\right), \mathrm{n}$-decane, formaldehyde and toluene were acquired from the Merck Company (Germany) and used as received.

\subsection{Instruments}

LXRD instrument (Bruker Nanostar U) with a wavelength of $\lambda=0.1542 \mathrm{~nm}$, a voltage of $45 \mathrm{kV}$, and a flow of $100 \mathrm{~mA}$ was utilized to record the XRD patterns. The range of the collection was between $2 \theta$ of $0.02^{\circ}$ and $3.5^{\circ}$ at a scanning rate of $0.05 \% \mathrm{~min}$. The thermal stability of samples in nitrogen atmosphere from the ambient temperature to $800^{\circ} \mathrm{C}$ was investigated by the TGA instrument (model = STA503 TA, Hullhorst, Germany). The surface morphology of the NCs was observed by FE-SEM (HITACHI, S-4160). TEM images were taken using a Philips CM120 (Eindhoven, Netherland).

\subsection{Synthesis of mesoporous carbon FDU-15}

Triblock copolymer (P123) and phenol/formaldehyde were used as a template and carbon precursor, respectively to synthesize the mesoporous carbon FDU-15 [23]. Briefly, at first, the exact amount of phenol (21 mmol) and formaldehyde solution ( $7 \mathrm{~mL}, 40 \mathrm{wt} \%$ ) was dissolved in $5 \mathrm{~mL}$ of $0.1 \mathrm{M} \mathrm{NaOH}$. After that, $11 \mathrm{mmol}$ of $\mathrm{n}$-decane was poured into the solution of $3.2 \mathrm{~g} \mathrm{P} 123$ in $50 \mathrm{~mL}$ of water. The mixture was continuously stirred for $5 \mathrm{~h}$ at $40^{\circ} \mathrm{C}$. After the addition of $60 \mathrm{~mL}$ of the phenol/formaldehyde solution, a white mixture was obtained. The color of the mixture changed to dark red as it was stirred for about $3 \mathrm{~h}$ at $65^{\circ} \mathrm{C}$ and it even became darker after the process continued overnight at the same temperature. The rotation process was continued for the next three days and then the solution was stirred for an additional $24 \mathrm{~h}$ at $70^{\circ} \mathrm{C}$. The resulting sediments were collected and calcined in the furnace at $350^{\circ} \mathrm{C}$. 


\subsection{Preparation of $\mathrm{m}-\mathrm{FDU}$ using $\mathrm{KH}-550$ as a modifier}

At first, $0.085 \mathrm{~g}$ of the dried FDU-15 were mixed with $10 \mathrm{~mL}$ of $\mathrm{KH}-550(0.004 \mathrm{v} / \mathrm{v})$ in ethanol and let stir for 10 min. Then, the resultant FDU-15 suspension was sonicated for $1 \mathrm{~h}$ with the power of $70 \mathrm{~W}$. Subsequently, the sonicated suspension was refluxed for $3 \mathrm{~h}$ at the ambient temperature and then separated using a filter paper and washed thoroughly with ethanol to remove the additional $\mathrm{KH}-550$ and then dried for overnight at $60^{\circ} \mathrm{C}$.

\subsection{In-situ preparation of PMMA/m-FDU-15 NCs}

The following path was used to synthesize the PMMA-m-FDU NCs: $\mathrm{m}$-FDUs were added to $5 \mathrm{~mL}$ of toluene and stirred and sonicated simultaneously in an ultrasonic bath for $2 \mathrm{~h}$. After that, the $\mathrm{m}$-FDU solution in toluene and $0.03 \mathrm{~g}$ of benzoyl peroxide were added to the $10 \mathrm{~mL}$ of MMA $(0.1 \mathrm{~g} / \mathrm{mL}$ toluene $)$ and refluxed for $6 \mathrm{~h}$ and then sonicated for $2 \mathrm{~h}$. Finally, the solutions were poured into a clean and smooth aluminum foil and let it dried overnight at $30^{\circ} \mathrm{C}$.

\section{Results And Discussion}

\subsection{Importance of substances, chemical agents, and methods}

Using PMMA as a pure polymer would not be practical in most applications because of its vulnerability and its low thermal stability. One of the simplest solutions to improve these defects is the incorporation of fillers into the polymer matrix $[24,25]$. Considering that every filler has its own properties, the polymer can obtain those properties as well. For example, if a filler possesses thermal resistance or bioactivity, the resulting NC would have these abilities too [26]. FDU-15 depicts a significant surface/volume ratio and also a resistance to heat and temperature, therefore, incorporating this filler into the polymer matrix can result in an NC with improved thermal stability and the ability to adsorb pollutants such as heavy metals and dyes in the aqueous solution [27]. Moreover, it has been suggested that for maximizing the interaction between fillers and the functional group of the polymer matrix, some modifiers with at least two functional groups might become handy [28]. Also, ultrasonic irradiation can produce very tiny bubbles in the size of a micrometer with tremendous temperature which facilitates the process of modification of FDU and $\mathrm{KH}-550$ and improves the interaction between the m-FDU and the ester functional group of the polymer which results in a homogenous NC with the least agglomeration [29]. Fig. 1 depicts a schematic representation of the possible interactions between FDU-15, KH-550, and PMMA matrix.

\subsection{FT-IR Spectroscopy}

The FT-IR spectra of both FDU-15 and m-FDU are shown in Fig. 2. A significant absorption band in the $1605 \mathrm{~cm}^{-1}$ and $1450 \mathrm{~cm}^{-1}$ which belong to the carbon materials confirms the successful synthesis of FDU-15 (Fig. 2a) [30]. The m-FDU also shows significant absorption peaks at $1060 \mathrm{~cm}^{-1}$ which is related 
to Si-O bonds [31]. The presence of both peaks at $1605 \mathrm{~cm}^{-1}$ and $1060 \mathrm{~cm}^{-1}$ proves the successful modification of FDU-15. Moreover, the presence of a peak nearly at $3200 \mathrm{~cm}^{-1}$ might be related to the $\mathrm{NH}$ stretching vibration of the $\mathrm{KH}-550$ modifier (Fig. 2b).

The FT-IR spectra of the PMMA and its related NCs are shown in Fig. 3. Two absorption bands in the area from $2900 \mathrm{~cm}^{-1}$ to $2950 \mathrm{~cm}^{-1}$ are related to asymmetric stretching vibrations of the aliphatic $\mathrm{C}-\mathrm{H}$ bond.

Also, a very weak absorption peak at $2850 \mathrm{~cm}^{-1}$ is attributed to the vibrational absorption of the $\mathrm{C}-\mathrm{H}$ bond in $\mathrm{CH}_{3}$. The carbonyl absorption stretching band of the ester functional group in the pure PMMA is observed at $1719 \mathrm{~cm}^{-1}$. A strong absorption band at the area around $1240 \mathrm{~cm}^{-1}$ to $1270 \mathrm{~cm}^{-1}$ belongs to the different C-O stretching modes. Significant changes in the fingerprint area $\left(400 \mathrm{~cm}^{-1}\right.$ to $\left.700 \mathrm{~cm}^{-1}\right)$ for the PMMA-m-FDU NCs are observable. These changes are the results of good interaction of $m$-FDU and the PMMA and successful preparation of the NCs. Also, the absorption of the Si-O band at $1080 \mathrm{~cm}^{-1}$ is another proof of successful fabrication of PMMA-m-FDU NCs [31].

\subsection{LXRD Diffraction Pattern}

The LXRD pattern of the FDU-15, m-FDU, and PMMA-m-FDU $1 \%$ and PMMA-m-FDU 3 wt $\%$ are shown in Fig. 4. For the FDU-15 a strong peak which is related to the (100) plane and the two weak peaks which are related to (101 and 200) prove the p6mm symmetry[32, 33] (Fig. 4d). The diffraction pattern of m-FDU also indicates that the same as that of FDU-15 with a tiny and slight shift to the lower angle. This shows that the modification process did not change the planes of the FDU-15, but instead, the pore structures have changed after the incorporation of KH-550 (Fig. 4c). The LXRD of the PMMA NCs still shows the peaks of the m-FDU, however, the more m-FDU is incorporated into the polymer, the intensity of the crystalline structure becomes weaker and this phenomenon could be explained by the fact that the polymer matrix interacts with the mesoporous structure and affects the regularity of the mesoporous mFDU in the long-range (Fig. 4. a, b).

\subsection{FE-SEM Micrographs}

Observation of the surface morphology of PMMA-m-FDU NCs was done by FE-SEM micrographs (Fig. 5). All the NCs show a unique dispersion of m-FDU mesoporous on their surface. Moreover, the more the amount of the m-FDU increases, the surface of the NCs becomes rougher. The incorporation of m-FDU mesoporous in the amounts of $1 \mathrm{wt} \%$ and $2 \mathrm{wt} \%$ has completely changed the morphology. The PMMA-mFDU $1 \mathrm{wt} \%$ and PMMA-m-FDU $2 \mathrm{wt} \%$ show some pores on their surfaces, therefore it can be deduced that the surface area of these NCs has been increased as the amount of the m-FDUs rises (Fig. 5b, c). However, the surface morphology of the PMMA-m-FDU 3 wt\% shows a considerable amount of agglomeration of m-FDU mesoporous on its surface (Fig. 5d). Therefore, the PMMA-m-FDU 2 wt\% with the highest surface area was chosen as an optimum for further investigation.

\subsection{TEM Images of the PMMA-m-FDU 2 wt\%}

The TEM images of the PMMA-m-FDU $2 \mathrm{wt} \%$ are shown in Fig. 6 . The water was chosen for achieving the maximum dispersion of the NCs. Fig. 6 a shows the uniform dispersion of the FDUs into the PMMA with 
an average size of $22 \mathrm{~nm}$ which has been obtained by its related histogram (SPSS. Statics 17.0). The uniform dispersion of the m-FDUs into the polymer matrix might be the consequence of the excellent interactions between the mesoporous material and the PMMA matrix. In addition, it can be seen easily that the structure of the $\mathrm{m}$-FDUs is well-ordered hexagonal arrays with long fringes which confirms the p6mm symmetry. Also, the incorporation of m-FDU into the PMMA has resulted in a little disorder on their surfaces due to the interactions between the polymer and the m-FDU (Fig. 6b).

\subsection{TGA analysis}

Figure 7 shows the TGA curves of the m-FDU and also PMMA-m-FDU NCs in the nitrogen atmosphere from the ambient temperature to $800^{\circ} \mathrm{C}$. Although several studies have proved that the pure FDU-15 has a negligible weight loss of around $5 \%$ even at the higher temperature, nearly $800^{\circ} \mathrm{C}$ [34], the $\mathrm{m}$-FDU shows a significant weight loss at far lower temperatures. As shown in Fig. 7d the m-FDU experiences several decomposition stages more than that of the pure FDU-15. The m-FDU has experienced (28 wt\%) weight loss in the 1 st decomposition state at around 100 to $320^{\circ} \mathrm{C}$. This is due to the water removal and the breakage of the physical bonds between the modifier and the FDU-15. Moreover, it has experienced another weight loss which is (7.3 wt\%) in the 2 nd decomposition stage at around 320 to $400^{\circ} \mathrm{C}$ because of the decomposition of the $\mathrm{KH}-550$ modifier. The last decomposition stage starts at $400^{\circ} \mathrm{C}$ and ends at $800^{\circ} \mathrm{C}$ with the $27 \mathrm{wt} \%$ weight loss with the rest mass of $37.3 \%$. These are related to the more stable materials which contain oxygen or the total combustion of the self-matrix (Fig. 7d).

The TGA thermographs of the PMMA (Fig. 7a) and PMMA NCs (Fig. 7b, c), however, show another trend. Although they illustrate a relatively similar trend, a deeper look reveals some exact changes. Generally speaking, as the amount of the m-FDU mesoporous increases into the matrix, the temperature of the decomposition states rises as well and they shift to the higher temperatures. For example, the $T_{d}$ (the temperature when there is a $10 \mathrm{wt} \%$ loss of the sample) of the PMMA was about $196^{\circ} \mathrm{C}$ and for the PMMA-m-FDU $1 \mathrm{wt} \%$ and PMMA-m-FDU $2 \mathrm{wt} \%$ was $300^{\circ} \mathrm{C}$ and $340^{\circ} \mathrm{C}$, respectively (Fig. 7a-c). Therefore, the incorporation of $\mathrm{m}$-FDU mesoporous has resulted in higher thermal stability for the NCs, because of the indigenous thermal stability of the FDU-15 and the excellent interaction of these mesoporous and the PMMA matrix. Moreover, the char yield (the total left over of the sample at $800^{\circ} \mathrm{C}$ ) of the pure PMMA was about 2\%, while for PMMA-m-FDU 1 wt\%'s and PMMA-m-FDU 2 wt\%'s were 4\% and 6\%, respectively.

\section{Conclusion}

A series of NCs based on the PMMA polymer and the FDU-15 were fabricated via the in-situ polymerization method with the aid of ultrasonic irradiation. During the process, the FDU-15 mesoporous structures were successfully synthesized by the use of P123 copolymer as the template and phenol/formaldehyde as a carbon precursor. Study of their functional groups, 2-dimensional hexagonal structure of these unique mesoporous, their morphology, and also their dispersion into the polymer matrix were confirmed and characterized by the FT-IR, LXRD, FE-SEM, and TEM analysis, respectively. The TGA measurement revealed that the NCs containing the m-FDU mesoporous possess a much higher thermal 
stability than that of the pure PMMA due to the better dispersion and better interactions between the mesoporous and the matrix. Among all the samples, the PMMA-m-FDU $2 \mathrm{wt} \%$ has possessed a higher surface area and better thermal stability than those of the other NCs.

\section{Declarations}

The authors are thankful to the Research Affairs Division of Isfahan University of Technology (IUT) Isfahan, I. R. Iran. The authors declare that they have no known competing for financial interests or personal relationships that could have appeared to influence the work reported in this paper.

\section{References}

1. G. Mohammadnezhad, P. Moshiri, M. Dinari, F. Steiniger, J. Iran. Chem. Soc. 2019 16, 716 (2019). https://doi.org/10.1007/s13738-019-01628-z 1491 ).

2. S. Hammani, A. Barhoum, M. Bechelany, Journal of Materials Science 2017 53:3 53, 1911 (2017). https://doi.org/10.1007/s10853-017-1654-9

3. M.A. Forte, R.M. Silva, C.J. Tavares, R.F.E. Silva, Polymers 2021, Vol. 13, Page 1346 13, 1346 (2021). https://doi.org/10.3390/polym13081346

4. F. McGrath, J. Qian, K. Gwynne, C. Kumah, D. Daly, C. Hrelescu, X. Zhang, D.M. O'Carroll, A. Louise, Bradley, Appl. Surf. Sci. 537, 147892 (2021). https://doi.org/10.1016/j.apsusc.2020.147892

5. S.B. Aziz, O.G. Abdullah, M.A. Brza, A.K. Azawy, D.A. Tahir, Results in Physics 15, 102776 (2019). https://doi.org/10.1016/j.rinp.2019.102776

6. T. Bubmann, A. Seidel, V. Altstädt, Polymers 2019, Vol. 11, Page 2070 11, 2070 (2019). https://doi.org/10.3390/polym11122070

7. Y.H. Li, X.Y. Shang, Y.J. Li, Mater. Lett. 270, 127744 (2020). https://doi.org/10.1016/j.matlet.2020.127744

8. G. Singh, S. Santhanakrishnan, Materials Technology, (2021) https://doi.org/10.1080/10667857.2021.1978640 (2021)

9. Y. Xu, D. Li, J. Shen, S. Guo, H.J. Sue, Polymer 182, 121829 (2019). https://doi.org/10.1016/j.polymer.2019.121829

10. Y. Xu, J. Qin, J. Shen, S. Guo, K. Lamnawar, Wear 486-487, 204069 (2021). https://doi.org/10.1016/j.wear.2021.204069

11. L. Liu, W.K. Meng, L. Li, G.J. Xu, X. Wang, L.Z. Chen, M.L. Wang, J.M. Lin, R.S. Zhao, Chem. Eng. J. 369, 920 (2019). https://doi.org/10.1016/j.cej.2019.03.148

12. D. Gang, Z. Uddin Ahmad, Q. Lian, L. Yao, M.E. Zappi, Chem. Eng. J. 403, 126286 (2021). https://doi.org/10.1016/j.cej.2020.126286

13. G. Mohammadnezhad, M. Dinari, R. Soltani, Z. Bozorgmehr, Appl. Surf. Sci. 346, 182 (2015). https://doi.org/10.1016/j.apsusc.2015.04.005 
14. D. Gang, Z. Uddin Ahmad, Q. Lian, L. Yao, M.E. Zappi, Chem. Eng. J. 403, 126286 (2021). https://doi.org/10.1016/j.cej.2020.126286

15. D.D. Eslek Koyuncu, M. Okur, Sep. Purif. Technol. 257, 117657 (2021). https://doi.org/10.1016/j.seppur.2020.117657

16. D. Feng, P. Li, Y. Feng, Y. Yan, X. Zhang, Microporous Mesoporous Mater. 310, 110631 (2021). https://doi.org/10.1016/j.micromeso.2020.110631

17. R. Wu, Q. Ye, K. Wu, H. Dai, J. Environ. Sci. 103, 172 (2021). https://doi.org/10.1016/j.jes.2020.10.014

18. M. Dinari, G. Mohammadnezhad, A. Nabiyan, Colloid and Polymer Science 2015 293:5 293, 1569 (2015). https://doi.org/10.1007/s00396-015-3556-1

19. S.R. Churipard, K.S. Kanakikodi, S.P. Maradur, ACS Symposium Series 1359, 483 (2020). https://doi.org/10.1021/bk-2020-1359.ch016

20. G. Mohammadnezhad, M. Dinari, R. Soltani, Z. Bozorgmehr, Appl. Surf. Sci. 346, 182 (2015). https://doi.org/10.1016/j.apsusc.2015.04.005

21. L. Sun, J. Huang, H. Liu, Y. Zhang, X. Ye, H. Zhang, A. Wu, Z. Wu, J. Hazard. Mater. 358, 10 (2018). https://doi.org/10.1016/j.jhazmat.2018.06.040

22. S. Mallakpour, M. Okhovat, Int. J. Biol. Macromol. 175, 330 (2021). https://doi.org/10.1016/j.jibiomac.2021.01.210

23. F. Zhang, Y. Meng, D. Gu, Z. Yan, B. Chen, Tu, D. Zhao, Chemi Mater., 18, 22, (2006). https://doi.org/10.1021/cm061400+

24. J. Zhu, C. Abeykoon, N. Karim, Int. J. Lightweight Mater. Manuf. 4, 370 (2021). https://doi.org/10.1016/j.ijlmm.2021.04.003

25. C. Bacali, M. Badea, M. Moldovan, C. Sarosi, V. Nastase, I. Baldea, R.S. Chiorean, M. Constantiniuc, Materials 2019, Vol. 12, Page 2335 12, 2335 (2019). https://doi.org/10.3390/ma12142335

26. G. Mohammadnezhad, A. Keikavousi Behbahan, J. Iran. Chem. Soc. 2020 17, 617 (2020). https://doi.org/10.1007/s13738-020-01864-8 1259).

27. G. Mohammadnezhad, P. Moshiri, M. Dinari, F. Steiniger, J. Iran. Chem. Soc. 2019 16, 716 (2019). https://doi.org/10.1007/s13738-019-01628-z 1491 ).

28. S. Fu, Z. Sun, P. Huang, Y. Li, N. Hu, Nano Mater. Sci. 1, 2 (2019). https://doi.org/10.1016/j.nanoms.2019.02.006

29. X. Dong, X. Liang, Y. Zhou, K. Bao, D.E. Sameen, S. Ahmed, J. Dai, W. Qin, Y. Liu, Int. J. Biol. Macromol. 177, 135 (2021). https://doi.org/10.1016/j.ijbiomac.2021.02.125

30. K.A. Trick, T.E. Saliba, Carbon 33, 1509 (1995). https://doi.org/10.1016/0008-6223(95)00092-R

31. J. Kathi, K.Y. Rhee, Journal of Materials Science 2007 43:1 43, 33 (2007). DOI https://doi.org/10.1039/B706695C

32. Y. Meng, D. Gu, F. Zhang, Y. Shi, H. Yang, Z. Li, C. Yu, B. Tu, D. Zhao, Angew. Chem. Int. Ed. 44, 7053 (2005). https://doi.org/10.1002/anie.200501561 
33. Z. Wu, P.A. Webley, D. Zhao, Langmuir 26, 10277 (2010). https://doi.org/10.1021/la100455w

34. N. Suzuki, S. Kiba, Y. Kamachi, N. Miyamoto, Y. Yamauchi, J. Mater. Chem. 21, 5338 (2011). DOI https://doi.org/10.1039/C0JM03767B

\section{Figures}

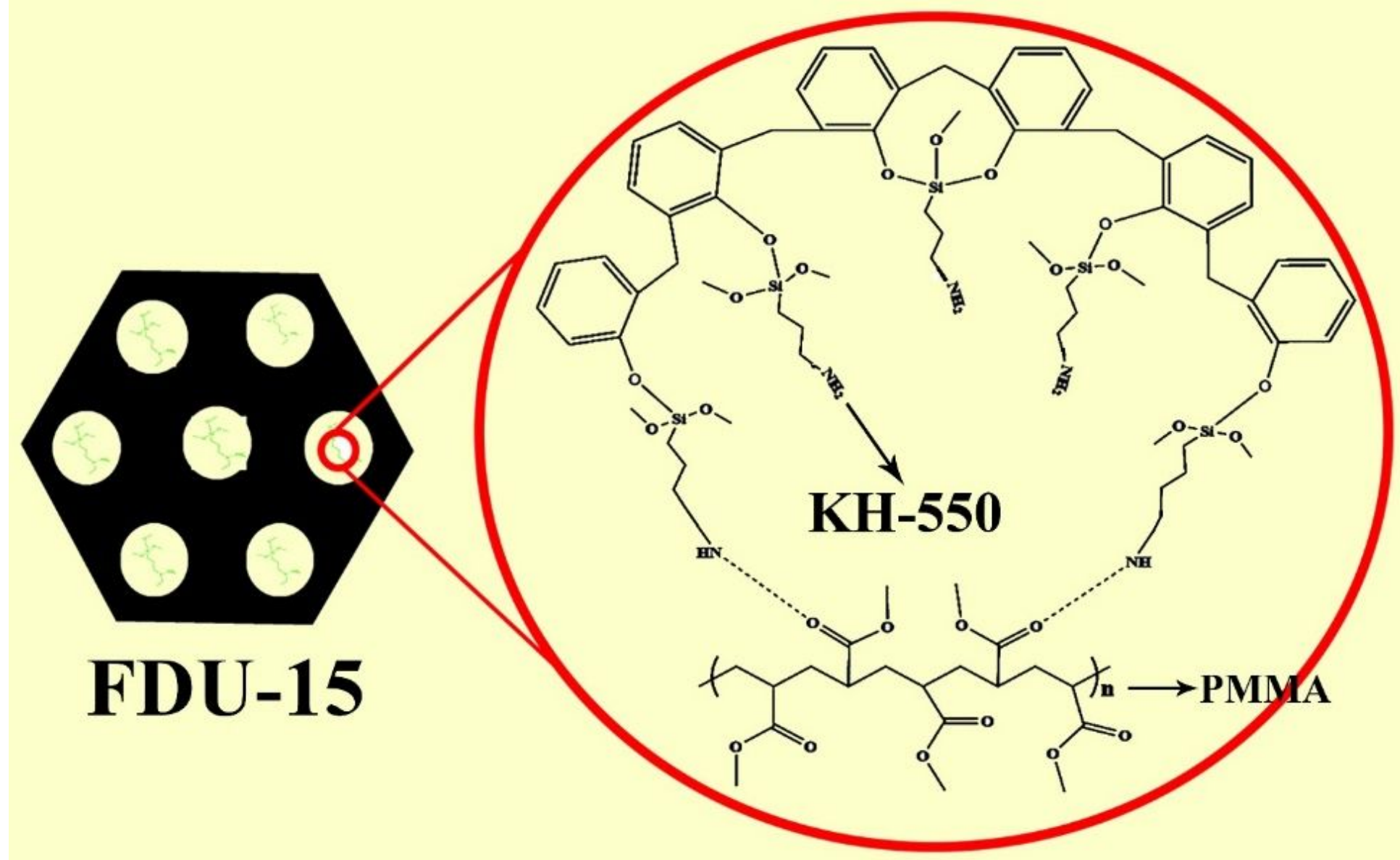

Figure 1

the possible interactions between FDU-15 and PMMA matrix 


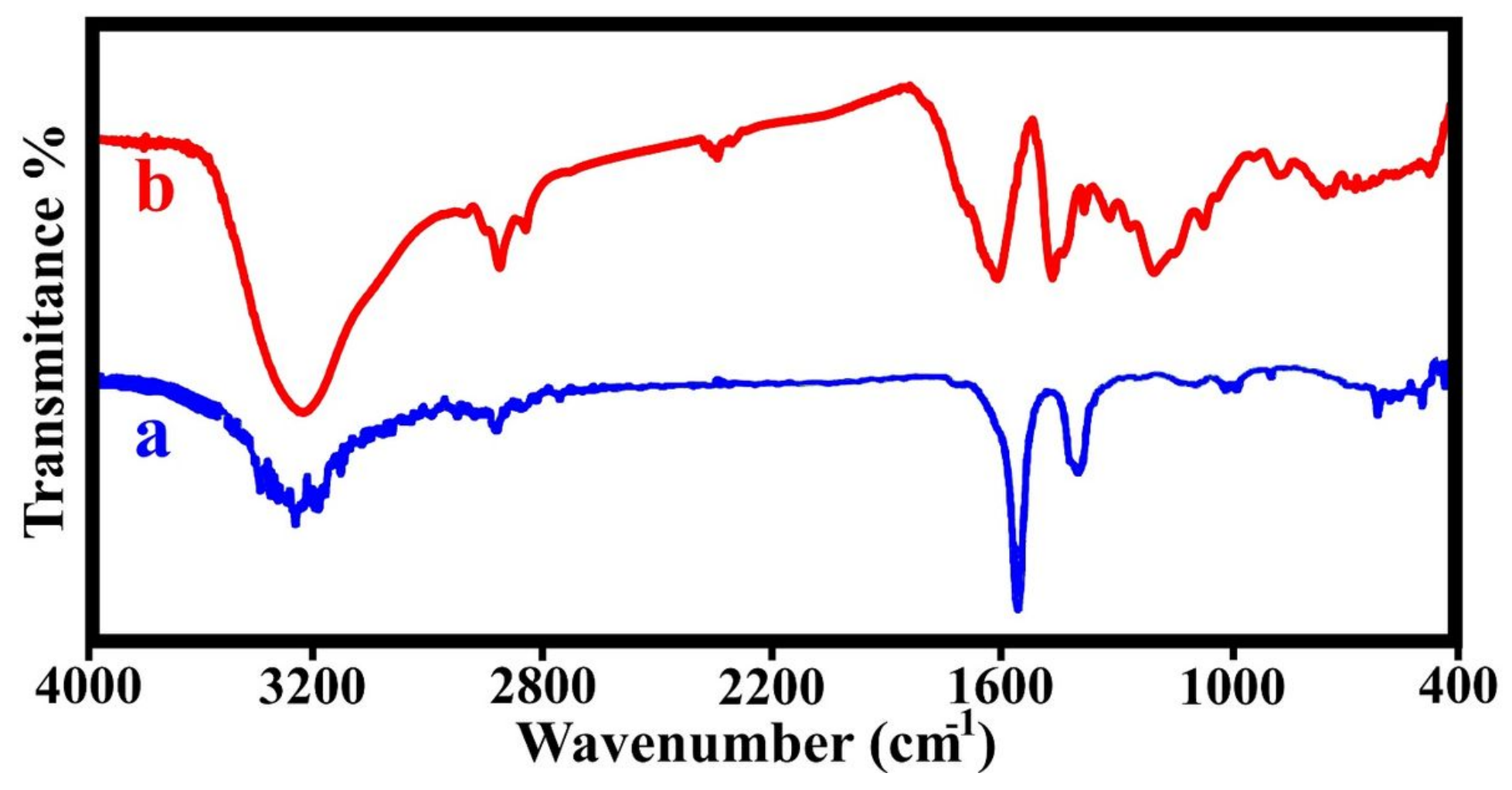

Figure 2

The FT-IR spectra of a) FDU-15 and b) m-FDU 


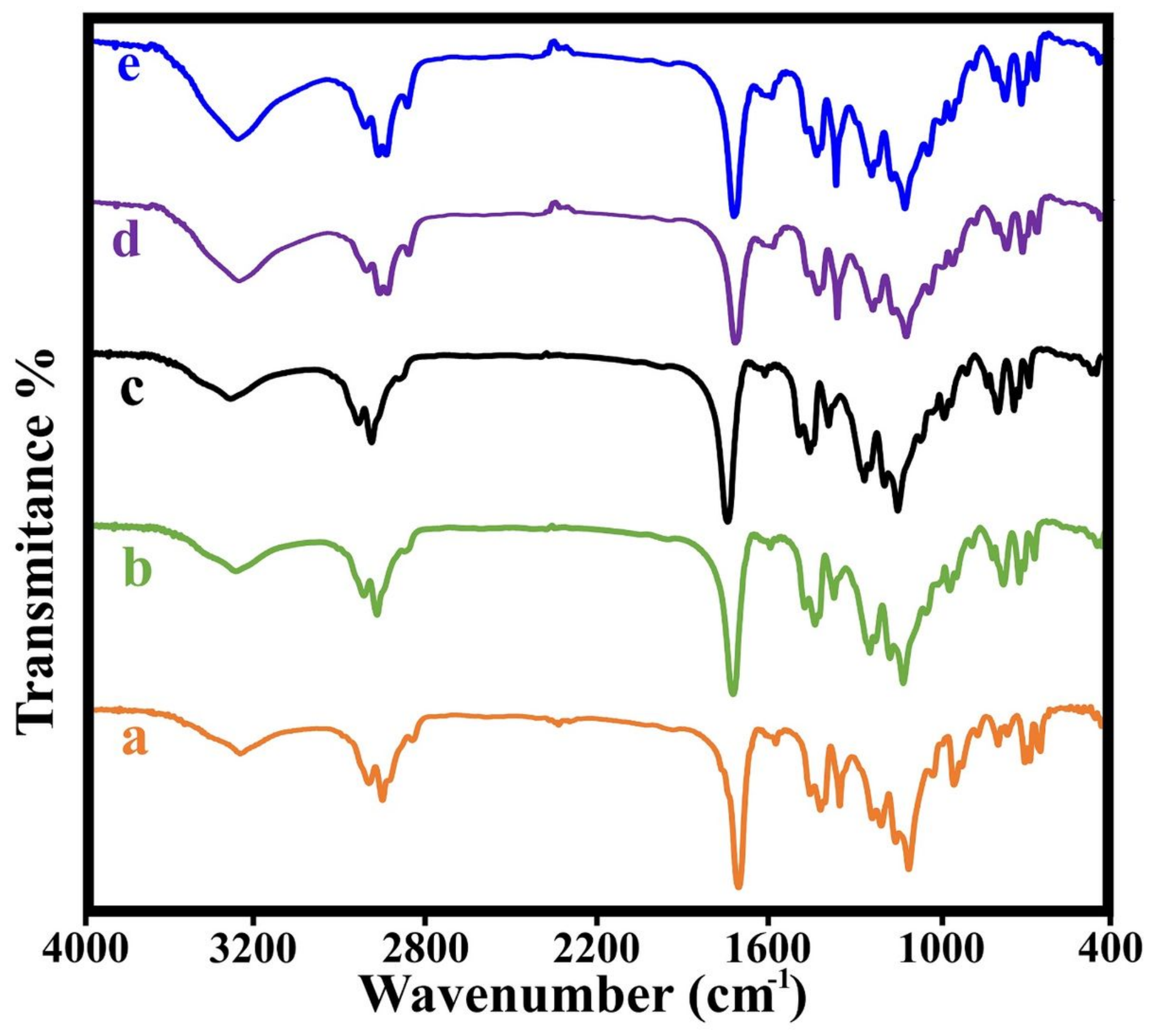

Figure 3

The FT-IR spectra of a) PMMA, b) PMMA-m-FDU 0.5 wt $\%$, c) PMMA-m-FDU 1 wt $\%$, d) PMMA-m-FDU 2 wt $\%$ and e) PMMA-m-FDU 3 wt\% 

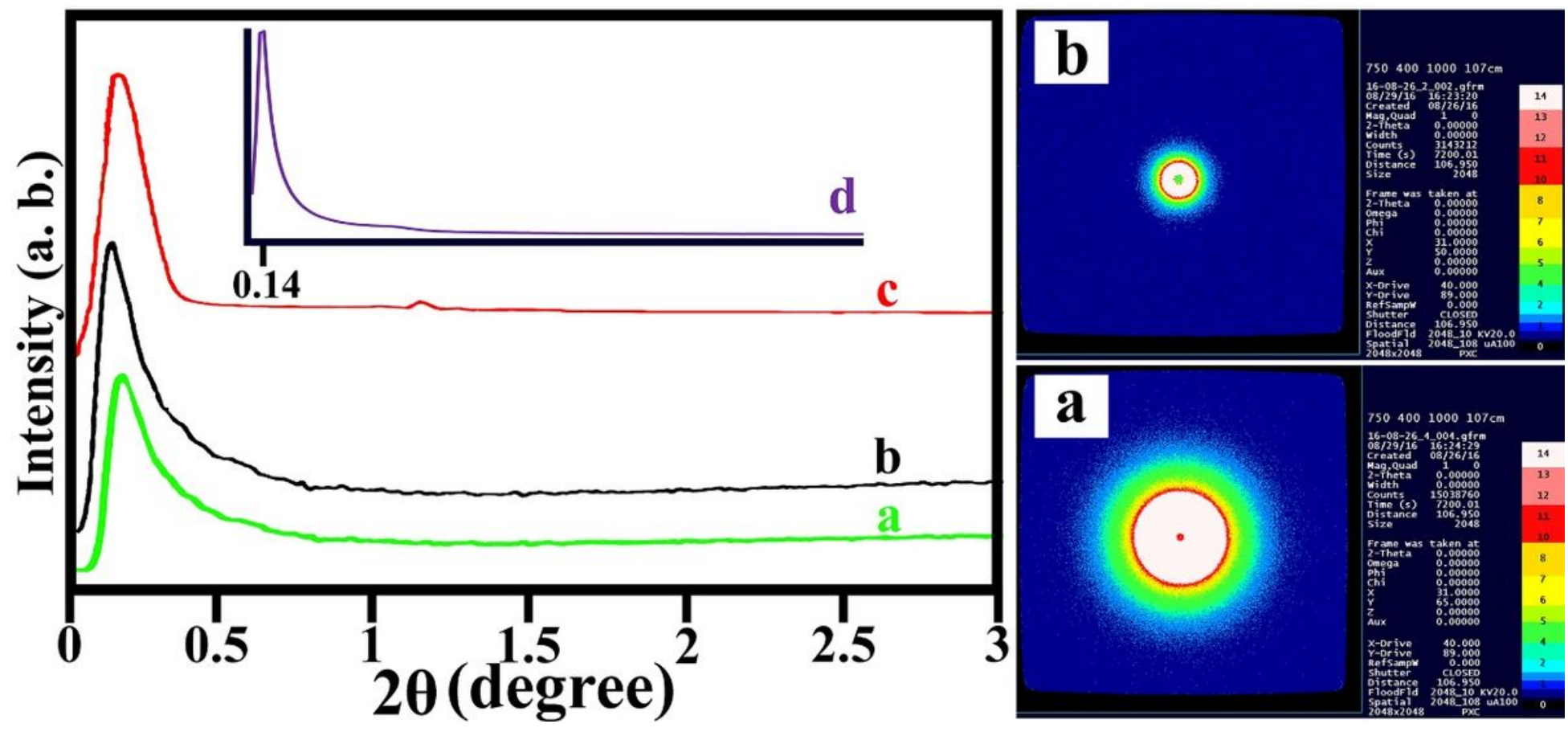

Figure 4

The LXRD pattern of the a) PMMA-m-FDU 3\%, b) PMMA-m-FDU 1\%, c) FDU-15 and d) m-FDU 


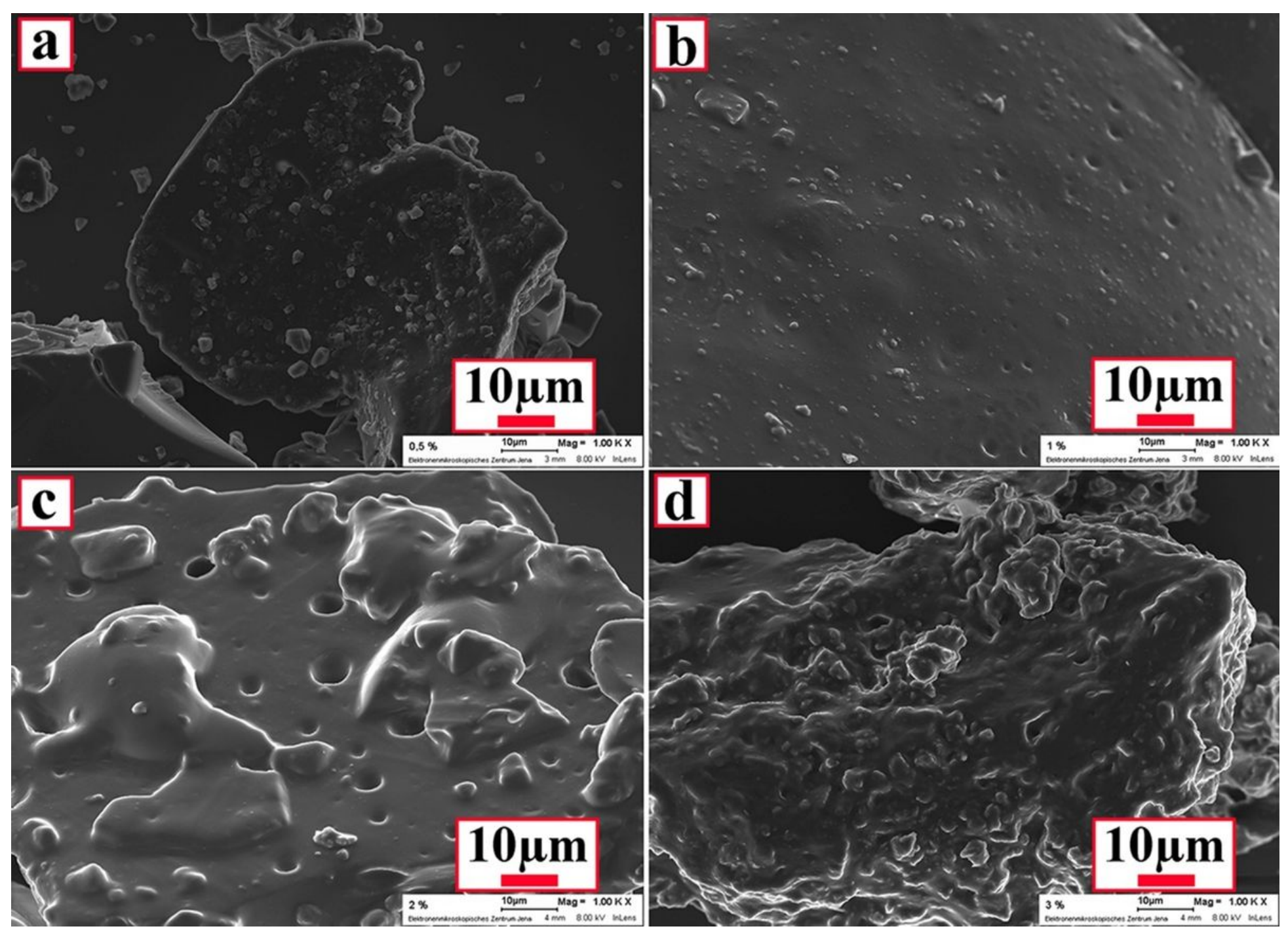

Figure 5

FE-SEM Micrographs of a) PMMA-m-FDU $0.5 \mathrm{wt} \%$, b) PMMA-m-FDU $1 \mathrm{wt} \%$, c) PMMA-m-FDU $2 \mathrm{wt} \%$ and d) PMMA-m-FDU 3 wt\% 

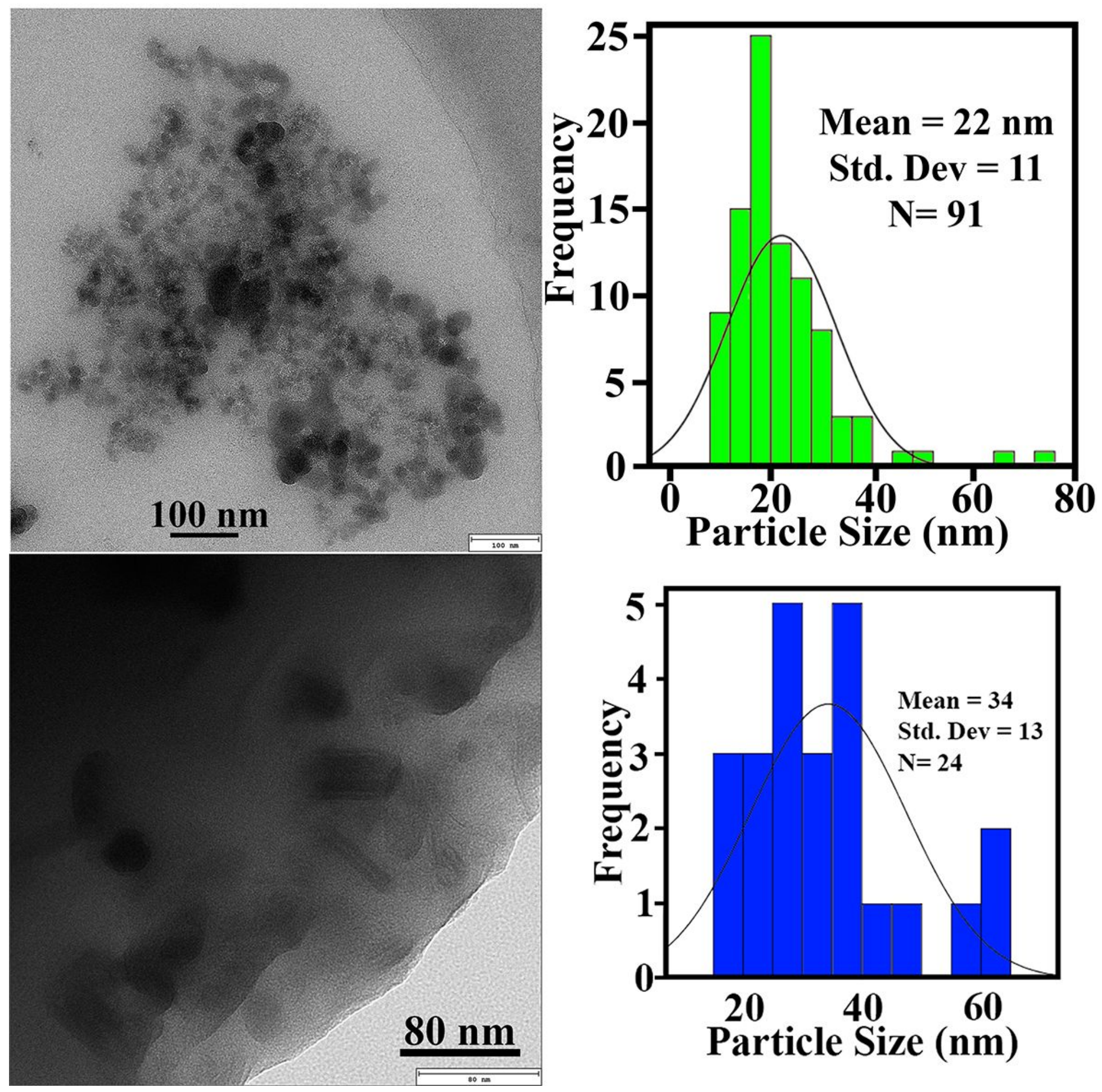

Figure 6

The TEM images of the a) PMMA-m-FDU 2 wt\% (100 nm) and b) PMMA-m-FDU 2 wt\% (80 nm) 


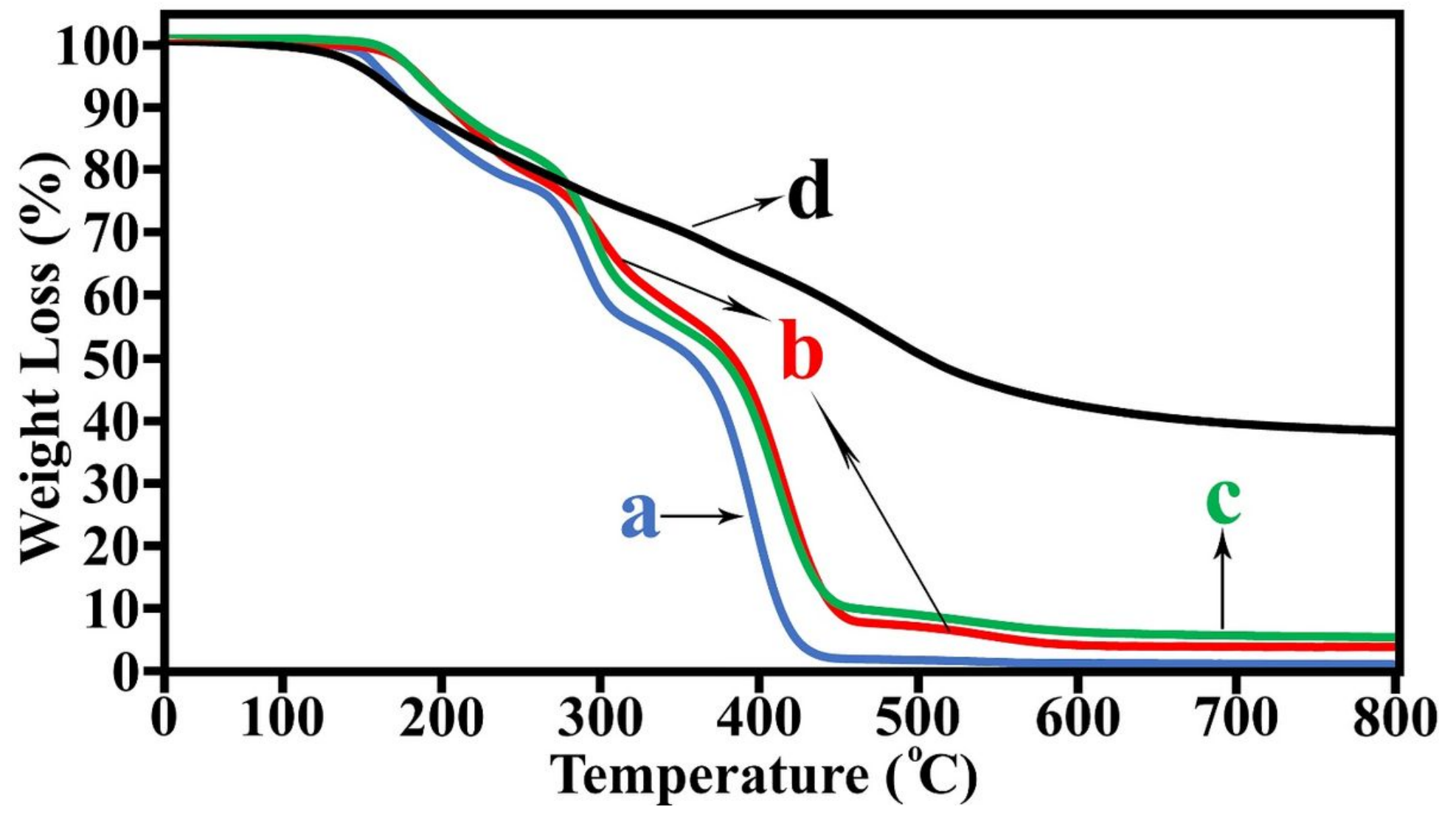

Figure 7

The TGA thermographs of a) pure PMMA, b) PMMA-m-FDU 2 wt $\%, c)$ PMMA-m-FDU 1 wt $\%$ and d) $m$-FDU 